\title{
The Direction Change Concept for Reticulospinal Control of Goldfish Escape
}

\author{
Mark B. Foreman ${ }^{\mathrm{a}}$ and Robert C. Eaton \\ Center for Neuroscience and Department of Biology, University of Colorado at Boulder, Boulder, Colorado 80309-0334
}

This is an analysis of whether biomechanical or kinematic variables are controlled by descending reticulospinal commands to the spinal cord during escape responses (C-starts) in the goldfish. We studied how the animal contracted its trunk musculature to orient an escape trajectory. We used trunk EMG recordings as a measure of the reticulospinal output to the musculature and we simuitaneously gathered high-speed cinematic records of the resulting movements. We found that the escape trajectory is controlled by (1) the relative size of the agonist versus the antagonist muscle contractions on two sides of the body and (2) the timing between these contractions. We found no separate signal for forward propulsion (or force) apart from the initial stage 1 bending of the body. Rather, the neural specification of force is embedded in the commands to bend the body. Thus, our findings demonstrate the importance of the angular kinematic components, or direction changes, caused by the descending reticulospinal command.

This new direction change concept is important for two reasons. First, it unifies the diversity of $\mathbf{C}$-start movement patterns into a single and rather simple quantitative model. Second, the model is analogous to the systematic EMG and kinematic changes observed by others to underlie single joint movements of limbs in other vertebrates such as primates. As in these cases, the fish capitalizes on the mechanical properties of the muscle by setting the extent and timing of agonist and antagonist contractions. This, plus the fact that sensory feedback is likely to be minimal, may enable the animal to reduce the number of computational steps in its motor commands used to produce the escape response. Because horizontal body movements in fish are a fundamental vertebrate movement pattern produced by a highly conserved brainstem movement system, our findings may have general implications for understanding the neural basis of rapid movements of diverse body parts.

IKey words: Mauthner neuron, sensorimotor coordination, motor control, brainstem, biomechanics, EMG, kinematics, neuroethology, teleost fish]

\footnotetext{
Received Nov. 27, 1992; revised Mar. 8, 1993; accepted Mar. 10, 1993.

This work was supported by a grant to R.C.E. from the NIH (RO1-NS22621). We thank R. DiDomenico, H. S. Duffy, A. Guzik, R. M. Johnston, R. K. K. Lee, D. E. Sherwood, and S. A. Wallace for their insightful comments. We also thank N. V. Doonskaya, B. C. Jayne, and P. W. Webb for extensive discussions and for providing us with prepublication copies of their manuscripts. M. M. Woodruff and L. M. Reed provided skillful technical assistance.

Correspondence should be addressed to Dr. Robert C. Eaton, Center for Neuroscience, University of Colorado, Boulder, CO, 80309-0334.

Present address: Mental Health Research Institute, University of Michigan, 205 Zina Pitcher Place, Ann Arbor, MI 48109-0720.

Copyright (C) 1993 Society for Neuroscience 0270-6474/93/134101-13\$05.00/0
}

A major issuc in the neurophysiology of motor control is to determine the parameters of movement that are specified by the descending motor commands of a given system. The various possibilities include kinematic variables such as position or angle, biomechanical variables such as acceleration or force, combinations of kinematic and biomechanical variables, or more complex considerations such as modulation of length-tension curves (for examples, see Evarts, 1966; Stein, 1982; Feldman, 1986; Bizzi and Mussa-Ivaldi, 1989; Soechting and Flanders, 1991; Weeks et al., 1991; Fetz, 1992). The reticulospinal system is an integral descending network involved in producing descending commands for many kinds of movements requiring large muscles of the body (for review, see Peterson, 1984). Thus, a fundamental problem is to learn what movement parameters are coded by the descending commands of the reticulospinal system.

To address this issuc, wc studicd reticulospinal control of movements involving changes in body orientation in an animal model, the C-type escape response (or "C-start") of the goldfish, shown in Figure $1 A$. Because goldfish and other members of aquatic vertebrate classes have identified reticulospinal neurons (McClellan, 1986; Metcalfe et al., 1986; Lee and Eaton, 1991; Lee et al., 1993), it should be possible to analyze the reticulospinal commands in terms of individual neurons. Of the reticulospinal cells in the goldfish, the paired Mauthner neurons and associated networks are already known to be involved in producing the C-start (Eaton et al., 1991; Faber et al., 1991; Fetcho, 1991). Like reticulospinal cells in general, the Mauthner neurons receive multimodal sensory input and make mono- or disynaptic connections onto motoneurons in the head and spinal cord.

The normal range of C-start escape trajectories involves activation of not only one of the Mauthner neurons but also an unspecified number of other reticulospinal cells; together with the Mauthner neurons, we call these neurons the brainstem escape network. We have postulated that this network regulates the trajectory away from the approach of the stimulus (Eaton et al., 1991). Numerous lines of evidence show that the Mauthner cell is involved in controlling the initial angular deviation of the head as the fish turns from the stimulus (stage 1 turn, Fig. 1A). The kinematic measure of this turn, or stage 1 angle (Fig. $1 C$ ), is comparable to a position variable. We have suggested that a second command might separately control the stroke of the tail underlying forward propulsion, or biomechanical stage 2 (stage 2 propulsion, Fig. $1 \mathrm{~A}$; Eaton et al., 1988), since forward propulsion of the center of mass is an essential biomechanical component of the C-start (Webb, 1978, 1993). Thus, according to this hypothesis, position and force are separately specified by the brainstem escape network. The specific 
A

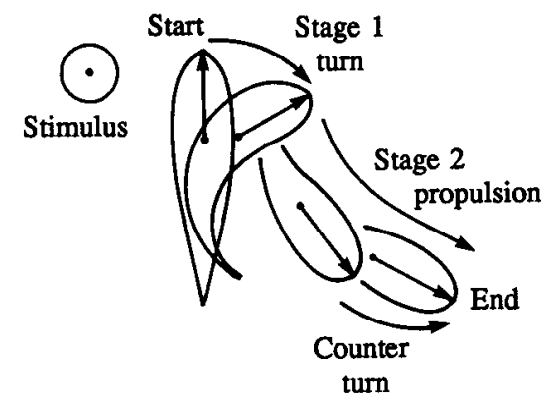

C

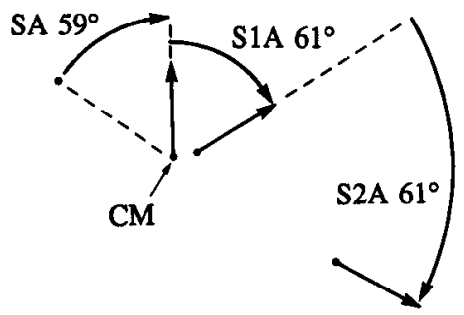

B
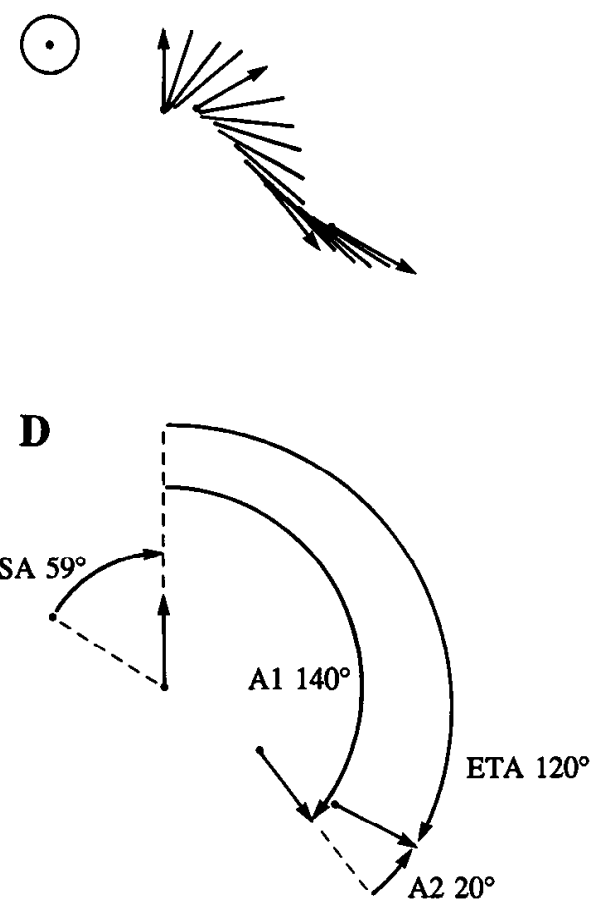

Figure 1. A goldfish escape response and its analysis. A, Response to a ball dropped into the water above the fish at the position marked stimulus. Biomechanically, the behavior consists of an initial turn away from the stimulus (stage l turn) followed by a forward propulsion (stage 2 propulsion), which may also be accompanied by a DC (counterturn). The last silhouette (end) shows the orientation and location, $50 \mathrm{msec}$ after the end of stage 1. $B$, Selected midlines calculated from the silhouettes constituting the response. $C$, Angular parameters used in our previous biomechanical model (Eaton et al., 1988). S1A is the number of degrees turned from the start position midline to the midline at the end of biomechanical stage 1 . Stage 1 ends and stage 2 begins when the center of mass $(C M)$ begins forward propulsion. This was detected when the center of mass became displaced more than the criterion distance of $0.5 \mathrm{~cm}$ (the relative distance is exaggerated in this diagram). Stage 2 angle $(S 2 A)$ is the difference in angular orientation between the midline at the start of stage 2 and the midline at $50 \mathrm{msec}$ after the end of stage 1 (this definition of S2A differs slightly from that in Eaton and Emberley, 1991). Although the present study did not use a stimulus with a known direction, the relationship of the SA to the angular kinematics is an important point in the Discussion. $D$. Angular parameters of the direction change model developed in this study. $A 1$ is the number of degrees turned in the initial orientation from the start position to the first DC (or to end of the behavior if there is no DC). $A 2$ is the number of degrees of the counterturn between $A 1$ and the end of the response, which determines the animal's apparent $E T A$. This is determined at $70 \mathrm{msec}$ after the start of movement. [Because $\mathrm{S} 2 \mathrm{~A}$ is defined over a $50 \mathrm{msec}$ interval, the sum of S1A plus $\mathrm{S} 2 \mathrm{~A}\left(122^{\circ}\right.$ in $C$ ) will be close to ETA $\left(120^{\circ}\right.$ in $\left.D\right)$ for cases such as this where stage 1 duration is about 20 msec.]

aim of the present experiments was to investigate this hypothesis and to learn how forward propulsion and trajectory are related to the contractions of the trunk musculature, which are a reflection of the descending reticulospinal commands. To perform this study, we used simultaneous EMG recordings and highspeed cinematic imaging.

We found that the C-start escape trajectory can be accounted for by the strength of the contractions on the two sides of the body and the interval between the two contractions. These contractions control the animal's angular orientation over time and its forward propulsion. We found no separate signal for forward propulsion (or force) apart from the initial stage 1 bending of the body. Thus, as predicted by Webb (1993), the important C-start parameters appear to be the variations in the timing and magnitude of directional control motions exerted by the body musculature. This conclusion simplifies the remarkable diversity of C-start kinematic patterns into a single conceptual framework. Moreover, our findings are analogous to systematic agonist and antagonist muscle contractions underlying rapid, singlejoint limb movements in other vertebrates, such as primates (Polit and Bizzi, 1978; Wallace, 1981; Schmidt et al., 1988; Sherwood et al., 1988; Doonskaya, 1992). Because of this, and the fundamental similarity of reticulospinal organization across vertebrates (Nissanov and Eaton, 1989; Lee et al., 1993), our findings may signify that the reticulospinal system uses common neurophysiological mechanisms for controlling rapid movements even in diverse vertebrates where the movement mechanics are different.

A preliminary report of this work was presented previously (Foreman and Eaton, 1990).

\section{Materials and Methods}

Animals and anesthesia. Goldfish (comet variety) of $10-13 \mathrm{~cm}$ standard length were obtained commercially. We maintained the fish on a 12/ $12 \mathrm{hr}$ light/dark photoperiod. They were supplied with a slow continuous flow of water from the municipal drinking watcr supply. This water was filtered through acid-washed carbon to remove chlorine and impurities. A commercial mixing valve automatically maintained the water at $16^{\circ} \mathrm{C}$. The water flowed through large standing tanks to buffer temperature changes and to allow the release of naturally compressed gases. Fish were fed four times per week on commercial trout chow. Under these conditions our goldfish consistently gained weight. For EMG recordings, fish were deeply anesthetized in 1:4000 MS-222 (ethyl- $m$-aminobenzoate; Sigma) prior to electrode implantation (described below). Following implantation, fish recovered in the testing arena for at least 15 min prior to behavioral testing. Data were obtained from $14 \mathrm{fish}$. 
Behavioral testing and acoustic stimulation. We studied C-starts of individual goldfish in an aquarium $(40 \mathrm{~cm} \times 40 \mathrm{~cm} \times 12.8 \mathrm{~cm}$ deep) with opaque walls. Fish were restricted within the aquarium by means of clear plastic partitions $(35.5 \mathrm{~cm} \times 35.5 \mathrm{~cm})$ to keep them in the field of view of the digital camera (see below). The arena was illuminated by diffuse light from above that produced a silhouette image of the fish as viewed by the camera (see below) from below the testing arena.

As described previously, escape responses were elicited by stimuli consisting of small, controlled, vertical movements of the entire test aquarium. Hydrophone recordings from various depths and locations within the test arena show that the vertical movement produces a complex sound field in the test arena (Eaton et al., 1988). The field intensity varies from a maximum near the bottom center to a minimum at the surface near the middle of the sides of the arena (Eaton et al., 1988). This variability is apparently perceived by the fish as a directional sound field. Stimuli were delivered when the camera showed that the fish swam into the center of the arena of its own accord. The fish could not see the experimenter who monitored its movements on a digital oscilloscope (see below). Starting at a subthreshold level, stimulus intensity was increased stepwise until a C-start was elicited (Eaton et al., 1988). The typical intertrial interval was about $15 \mathrm{~min}$. Trials were continued until an average of 12 responses had been obtained from each fish. Because the orientation of the fish was random with respect to the sound field, the fish produced a random distribution of escape trajectories (Eaton et al., 1988; see also Results).

Digital image capture and analysis. We recorded behavioral data at $500 \mathrm{frames} / \mathrm{sec}$ with a matrix camera. This is a digital machine vision device interfaced to a Compaq $386 / 20 \mathrm{E}$ computer that used several programs to capture the image and analyze the movement automatically. Basically, the system is designed to return 52 silhouette images of the fish at 2 msec intervals. Selected silhouettes are shown in the example in Figure 1A. For each of the silhouettes we computed the anterior body midline (Eaton et al., 1988). Selected midlines are shown in the example in Figure $1 B$. The movement of this line in space forms the basis of our kinematic measurements.

For some studies of continuous locomotion, body curvature is an appropriate measure of the underlying muscular contractions (Cohen, 1992). Our angular measurements are a function of overall curvature of the trunk. For our studies, midline angle is most appropriate because it has a straightforward relationship to the components underlying the escape trajectory that can be directly related to the attack angle of an aversive stimulus (Eaton and Emberley, 1991). In addition, because of the anterior stiffness of the body, little error is introduced by analyzing the anterior midline rather than body curvature.

Our digital analysis required the development of new kinematic parameters that could be related to underlying patterns of EMG activity. These angles are contrasted with our previous angular parameters in Figure $1, C$ and $D$. The new parameters, shown in Figure $1 D$, are derived from the sequence of turns that produce the apparent escape trajectory angle (ETA). This was operationally defined as the heading of the fish at $70 \mathrm{msec}$ after movement onset relative to the starting position of the fish (Eaton and Emberley, 1991). This interval is within the closing times of predatory attacks (Webb and Skadsen, 1980; Lauder, 1983) and is a temporal limit of our present analysis system. The ETA can be produced by a single unilateral turn to one side, or by an initial turn and counterturn, as shown in the example in Figure 1. These two turns are measured in degrees and, as indicated in Figure $1 D$, are referred to as angle $I(A 1)$ and angle $2(A 2)$, respectively. Thus, by definition,

$$
\mathrm{ETA}=A 1-A 2 .
$$

In our study these angles were determined automatically by the computer. By definition, in unilateral turns, $A 2=0$ and FTA $=A 1$

Our study concerned only the large angular components $(A 1$ and $A 2)$ contributing to ETA measurements at $70 \mathrm{msec}$. In about $7 \%$ of the trials there was an additional direction change (DC), $A 3$, which was opposite $A 2$ and near the end of the response. However, these small turns were too infrequent for separate statistical evaluation and were not incorporated into our data set. In cases with an $A 3$ turn, the effect of this was to cause $A 2$ to be slightly underestimated. This had little impact on our overall results, as evidenced by the angular calibration described below; moreover, the concept of a third turn would be easily accommodated by the model resulting from our findings.

In the interval since our initial study using digital imaging (Eaton et al., 1988), the system has undergone important technical refinements that permit the more sophisticated analyses of the present study. The first major modification involved applying smoothing algorithms to the midlines to eliminate spatial noise in the digital image. This produced midline representations that faithfully resemble those drawn by hand from filmed images. A second important modification involved an algorithm to determine more accurately the onset of forward propulsion, or stage 2 . This calculation employed a linear regression of the movement of the center of mass over time (see dashed line, Fig. 3B). The deviation of this regression from the $\mathrm{x}$ - (or time) axis defined stage 2 onset (see, e.g., S2, Fig. 3). Additionally, we required that this point had to move more than $0.5 \mathrm{~cm}$ from the point of rotation of the center of mass during stage 1 . This is about $4 \%$ of a body length and is a $33 \%$ improvement over our previous work (Eaton et al., 1988) and only $0.7 \%$ greater than measurements made by eye from projected images of cinefilm (Eaton and Emberley, 1991). The effect of this conservative criterion was to overestimate slightly the time to stage 2 onset, but it prevented false-positive assessments of stage 2 onset. As a measure of the accuracy of our automated angular measurements and calculations, we performed a linear regression on a plot of ETA versus $A 1$ minus $A 2$ for 162 responses. This plot resulted in the following regression:

$$
\mathrm{ETA}=0.99(A 1-A 2)+1.4^{\circ} \quad(p<0.001) .
$$

This demonstrates the precision of our digital analysis; Equation 2, calculated from empirical measurements, was within a few degrees of the theoretical ideal given by Equation 1 .

$E M G$ recording. Our study is based on the assumption that EMG recordings can be used as a low-pass-filtered version (Gottlieb et al., 1989) of the reticulospinal command to the motoneurons. Results from EMG recordings, however, depend highly on electrode configuration and placement (Loeb and Gans, 1986; Wainwright, 1989). Therefore, we tested a variety of electrode configurations and rostral-caudal placements to determine the method that best revealed the activity of a large portion of muscle mass involved in producing the body contractions associated with the C-start. Wide-field, bipolar, electrode wires (with an interpole distance of approximately $5 \mathrm{~mm}$ ) placed in the midcaudal trunk region yielded the most information about the muscle activity producing the contractions of the C-start and were the most similar to our earlier recordings (Eaton et al., 1988). This region, near the end of the body cavity, has a large muscle mass and is also the focus of maximal body curvature and muscle contraction during the period of peak caudal fin velocity (Williams et al., 1989). Refer to Wainwright (1983) for a further description of the structure of the trunk musculature in fishes.

We fabricated our EMG electrodes from insulated $75 \mu \mathrm{m}$ copper wires whose tips were delaminated by flaming and sanding the distal $1-2 \mathrm{~mm}$. Electrode tips were hooked and inserted, using syringe needles, into the dorsal white epaxial musculature two scale widths up from the posterior lateral line to a depth of $3-4 \mathrm{~mm}$. We used a suture to anchor the electrode leads to the skin just rostral to the dorsal fin. In this way, struggling movements or normal activity would place tension on the anchor and not on the inserted wires. We cabled multiple electrode wires together as a group by running the leads through a droplet of cyanoacrylate glue between the thumb and forefinger (George $V$. Lauder, personal communication). This aided handling of the wires as well as reduced knotting. EMG leads from the animal were of sufficient length not to restrain the movements of the fish.

We differentially amplified and band-passed the EMG signals from 300 to $3000 \mathrm{~Hz}$ by Grass P 15 preamplifiers, and then rectified the signals by in-line absolute value circuits. The rectified traces were digitized and stored in an oscilloscope for subsequent downloading to computer. Calculations of EMG integrals, rise times, latencies, durations, and averages were accomplished by programs developed in our laboratory. EMG recordings were obtained for all 165 trials that we cinematically recorded.

\section{Results}

\section{Characterization of the escape behavior}

The main issue in this study was to relate the pattern of EMG potentials to the kinematic and biomechanical parameters that determine the escape trajectory. The movement patterns can be complex even during the first $70 \mathrm{msec}$ of the escape. The complexity is illustrated by the 11 examples of Figure 2, which were chosen from several different animals to illustrate the wide range of possible escape trajectories and kinematic patterns that can occur. To compare different responses in this figure, we stan- 
Figure 2. Examples of the wide range of escape trajectories and the corresponding kinematic patterns that can occur in goldfish. Central fish outline represents the standardized position at the start of movement. Arrows emanating from the center of mass give the ETAs for the responses illustrated by the midline animations at the outside of the diagram. Arrow lengths are proportional to distance moved by the center of mass for each of the responses shown. The dots on the compass indicate the angular heading for each escape trajectory. The responses were chosen from a data set of 165 responses in 14 animals, and show that the goldfish is capable of achieving virtually any escape trajectory in the entire $360^{\circ}$ range. The diversity of the response patterns is related to the direction of the stimulus perceived by the fish and the orientation and distance of the fish to the walls of the test arena (Eaton and Emberley, 1991). For purposes of graphical consistency, all responses in this study are shown as though the initial turn was in the clockwise direction.

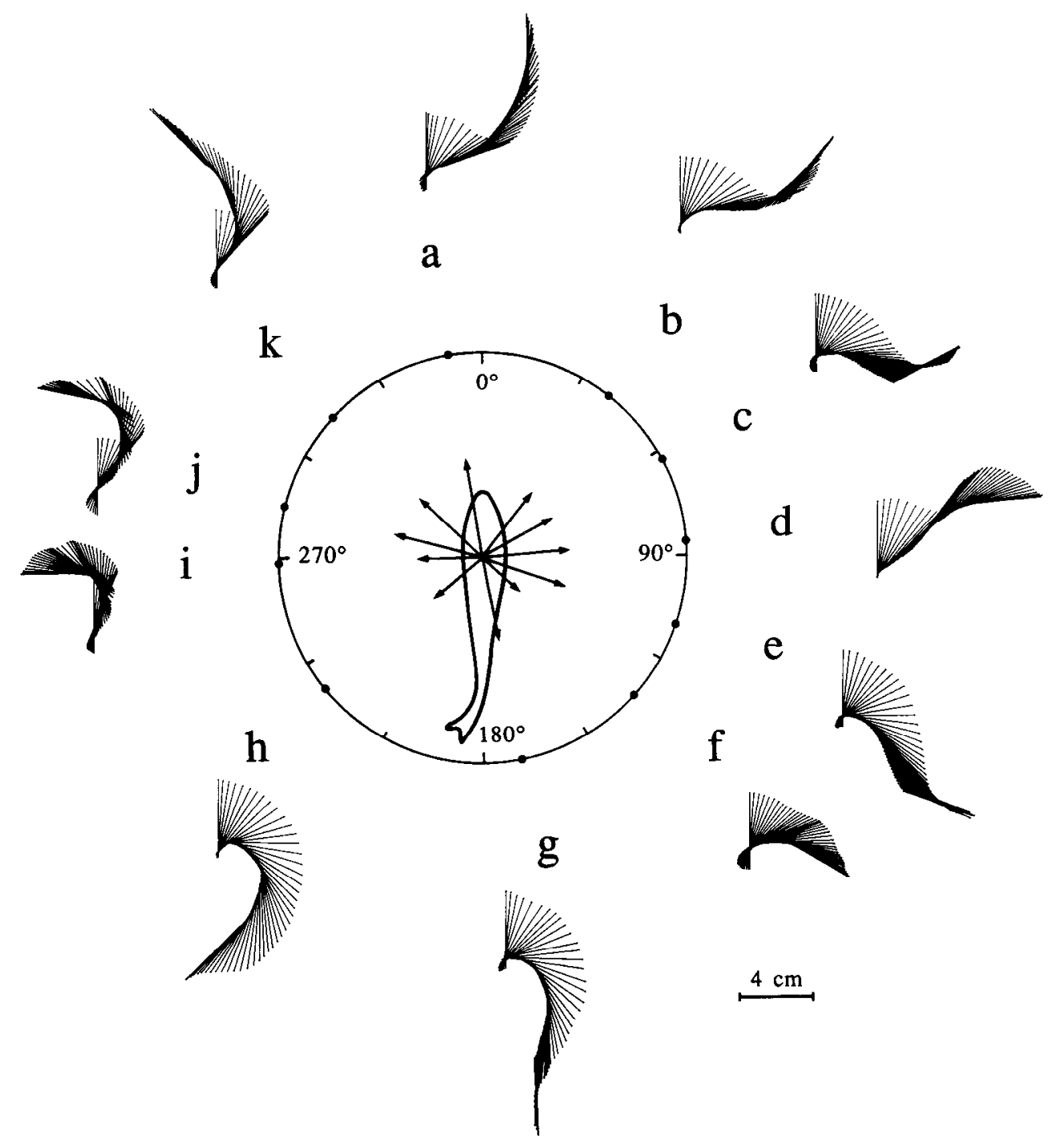

dardized the starting positions of all responses so that the fish had initial orientations of $0^{\circ}$ as illustrated by the central fish silhouette. For purposes of graphical consistency, all responses are shown as though they are in the clockwise direction. The escape trajectory headings for the 11 responses were arranged as arrows emanating from the center of the compass, which coincides with the animals' center of mass. Each arrow is in the direction of the individual escape trajectories relative to the starting orientation. These are indicated by dots on the surrounding compass. Figure 2 shows that within $70 \mathrm{msec}$ the fish oriented virtually anywhere in the $360^{\circ}$ horizontal plane around the starting position.

The midline animations arranged around the outside of the central compass in Figure 2 illustrate the kinematic patterns underlying this wide range of trajectories produced during the 11 responses. As defined in Figure 1, the responses consist of both an initial rotation of the midline, or stage 1 , and a forward propulsion, or stage 2 , which may also be accompanied by further turning movements. The initial rotation is caused by a body bend on the side opposite the perceived direction of the stimulus. This is the major side contraction. This contraction causes the head to deviate from the starting position to an orientation away from the stimulus (Eaton and Emberley, 1991). The sim- plest responses consist of a unilateral turn to one side (such as Fig. $2 f, h$ ). For one such response, the stage 1 rotation is plotted versus time as the solid line labeled "angle" in Figure $3 B$. This kind of response would occur when the stimulus is perceived by the fish as coming from the left side. Thirty-eight milliseconds after this fish started rotating, it began forward propulsion, or stage 2 (labeled "S2"), as indicated by the dashed line labeled "movement" in Figure $3 B$. Here, the angular heading at the transition between stage 1 and stage 2 was $57^{\circ}$. This parameter is known as stage 1 angle. (Please refer to Fig. $1 C$ for a further description of this parameter.) The escape trajectory was $119^{\circ}$ at $70 \mathrm{msec}$ after the start of movement.

For most responses illustrated in Figure 2, however, the escape trajectory was not produced by a unilateral bending. Rather, the escape was accomplished by varying the extent and onset time of a second contraction of the axial musculature on the side opposite the major side contraction. This minor side contraction resulted in a change in direction (DC) during the response. Some of the C-starts involved a reversal in direction (Fig. $2 a, i-k$ ), whereas others made relatively small corrections in trajectory (Fig. $2 e, g$ ). The onset times of the DCs could be either relatively early in the response (Fig. $2 i$ ) or relatively late (Fig. 2e). Thus, DCs were defined as responses where the angular 
A

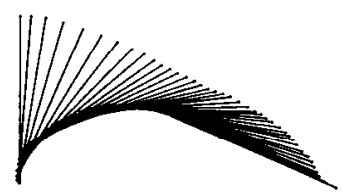

B

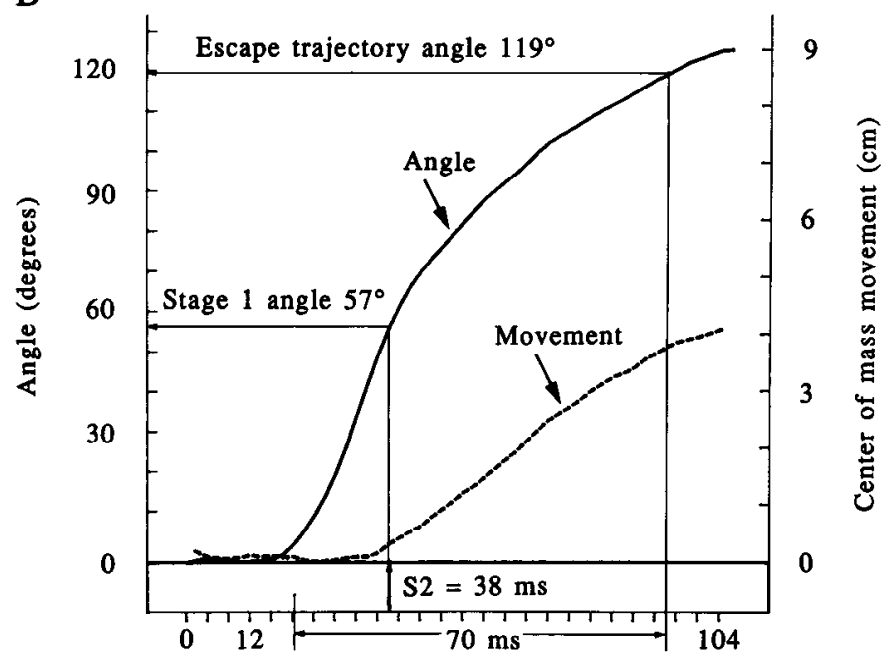

Time after start (ms)

Figure 3. Analysis of a C-start with a unilateral turn to the right. $A$, Midline animation of response. $B$, Angular and movement data for the response in $A$. Stage 2 starts at $38 \mathrm{msec}$, which ends stage 1 with an angle of $57^{\circ}$. This fish achieved an ETA of $119^{\circ}$. In this case $A 1$ was equal to the ETA.

velocity either stabilized at zero, or reversed and became negative relative to the initial movement. In our entire data set, $42 \%$ of the responses had DCs.

Figure 4 gives the quantitative analysis of a response involving a DC. Such a turn would be produced in response to a stimulus approaching the fish from behind (Eaton and Emberley, 1991). In this response, the DC occurred $8 \mathrm{msec}$ after stage 2 onset and resulted in an escape trajectory of $18^{\circ}$ relative to the start orientation. The presence of $\mathrm{DC}$ required us to introduce two new angular parameters; $A 1$, the heading at the time of the DC, and $A 2$, the turn occurring between the DC and escape trajectory. Please refer to Figure $1 D$ for a further description of these parameters.

To summarize, the midline animations in Figures $2-4$ show that the wide range of $\mathrm{C}$-start trajectories is accomplished because of the ability of the fish to execute either unilateral turns of varying magnitude (Figs. $2 f, h ; 3)$ or turns containing a direction change at a variable time after the start of movement (Figs. $2 a-e, g, i-k ; 4)$. Thus, the entire range of movements is due to one or two turns that vary in magnitude and onset time. It is this variability that needs to be accounted for in models of the output of the descending reticulospinal command.

\section{Relationship between body muscle contractions and escape trajectory}

We previously identified two bursts of EMG activity that occurred sequentially in the musculature of the two sides of the
A

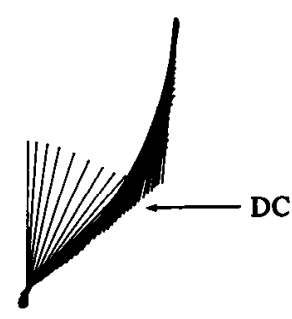

B

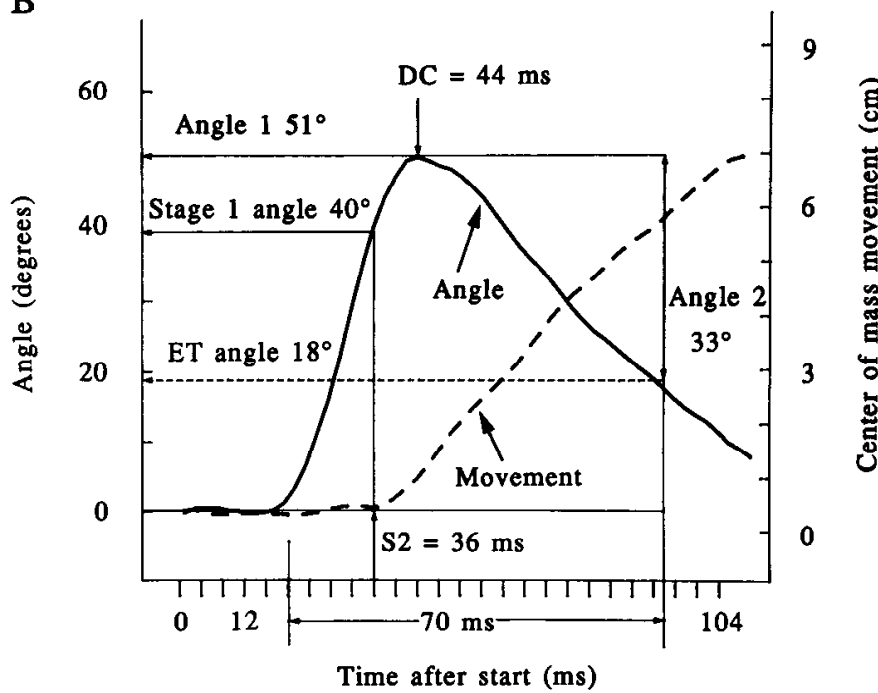

Figure 4. Analysis of a C-start with an initial right turn followed by a DC to the left. $A$ and $B$ same as Figure 3. In this response the DC occurred substantially later than stage 2 onset $(36 \mathrm{msec})$. Notice that the $E T$ angle is the algebraic sum of $A 1$ and $A 2$.

body during the C-start (Eaton et al., 1988). The size of the first of these EMGs, which we call the major side EMG, was significantly correlated with the extent of the angle achieved by the cnd of stage 1 . We did not attempt to relate the second, or minor side $E M G$, to any movement parameter. The minor side EMG was prominent, however, in responses having a $D C$ and we hypothesized that this EMG might reflect a contraction causing stage 2 propulsion (Eaton et al., 1988). If correct, the minor side EMG should correspond temporally to stage 2 onset with a delay equivalent to the muscle activation time. We would expect this interval to be as long as, or longer than, the $7 \mathrm{msec}$ muscle activation time following Mauthner cell firing (Eaton et al., 1981, 1988, 1991). The Mauthner cell activates white, fast-twitch muscle fibers (Fetcho, 1991) and should provide the shortest activation time. To investigate this issue, we next analyzed the functional consequences of the contraction reflected by the minor side EMG.

Contrary to expectation, onset of stage 2 is not related to the minor side EMG. This can be determined by careful analysis of EMG rccords, like that shown in Figure 5. Here we obtained bilateral, midtrunk EMG recordings with simultaneous matrix camera analyses of the C-start kinematics (the EMG records in Fig. $5 B$ have the same time scale as the kinematic analysis in Fig. $5 C$ ). As we previously reported for responses of this kind (Eaton et al., 1988), there were two prominent bursts of EMG activity. The first burst occurred on the right side of the bodythe major side (upper trace, Fig. $5 B$ ). This burst preceded the stage 1 orientation to the right by an interval of $7 \mathrm{msec}$ (indicated 


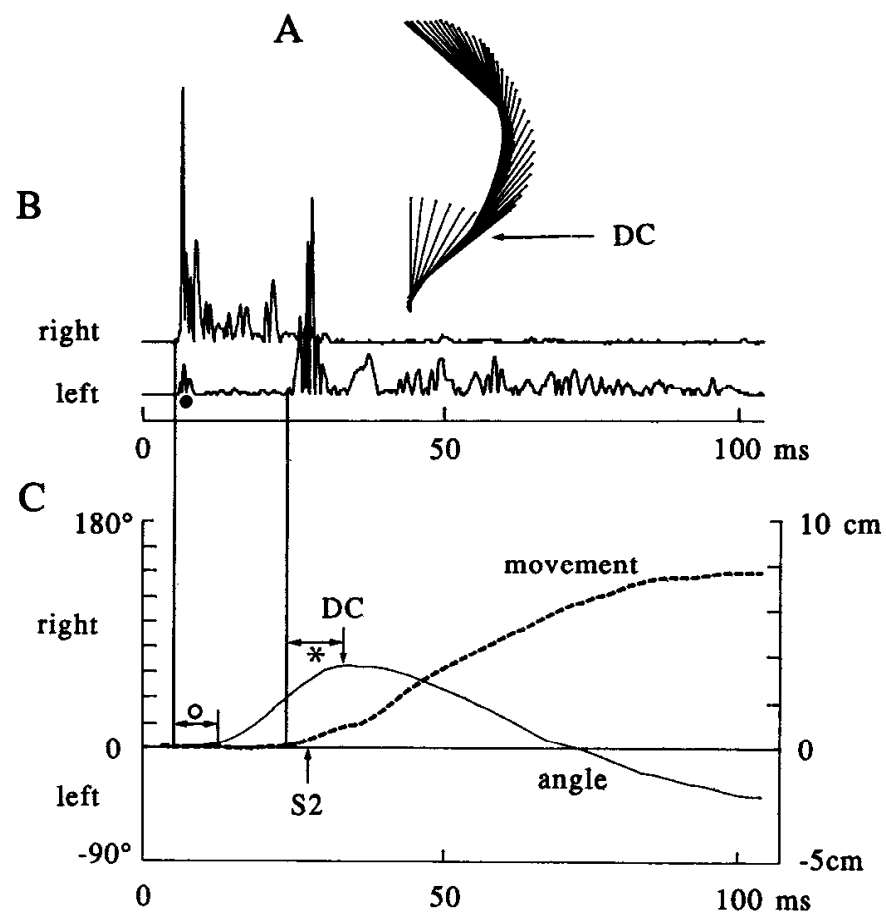

Figure 5. Combined EMG and kinematic data from a response with a DC. $A$ and $C$ are as in Figures 3 and 4. $B$, Bilateral trunk EMGs. This example shows that the minor side EMG is associated with a DC rather than forward propulsion of the center of mass. The major side EMG on the right (upper trace) accompanies a contraction that produced a right turn. This was followed by a minor side EMG on the left (lower trace) accompanying a contraction that caused a DC (DC in $A$ and $C)$. The interval from the minor side EMG onset to center of mass propulsion is too short $(3.5 \mathrm{msec})$ to have led to the center of mass propulsion ( $S 2$ and dashed line in $C$ ). The relatively large minor side contraction resulted in negative escape trajectory heading to the left (solid line in $C$ ).

by the open circle), comparable to the expected muscle activation time for Mauthner-initiated C-starts.

The second large burst of muscle activity occurred on the left or minor side (lower trace, Fig. $5 B$; below we discuss the small burst indicated by the dot that occurs in many EMG recordings of this type). The relative timing between the minor side EMG and the kinematics rules out the activation of stage 2 propulsion by the minor side EMG burst. Notice that the minor side EMG burst was nearly coincident with the deviation of the movement record (dashed line) from the baseline in Figure 5. As automatically determined by our computer algorithm, the interval between the minor side EMG and movement onset of stage 2 (S2, Fig. $5 C$ ) was only $3.5 \mathrm{msec}$. This interval is only half the muscle activation time associated with Mauthner firing and is therefore too short to lead us to believe that the minor side EMG is associated with stage 2 onset. Rather, the minor side EMG appears to correlate with the DC to the left (DC, Fig. $5 C$ ). In fact, the minor side burst preceded the DC by $9.6 \mathrm{msec}$ (asterisk), a reasonable value for thinking that the minor side EMG leads to the DC.

Thus, the data in Figure 5 seem to contradict the initial hypothesis. Examples of other responses corroborate this conclusion. For example, a minor side EMG was absent in responses in which the animal made a large unilateral turn and did not change direction afterward. This is illustrated in Figure 6, in which there was a large EMG major side burst recorded in the
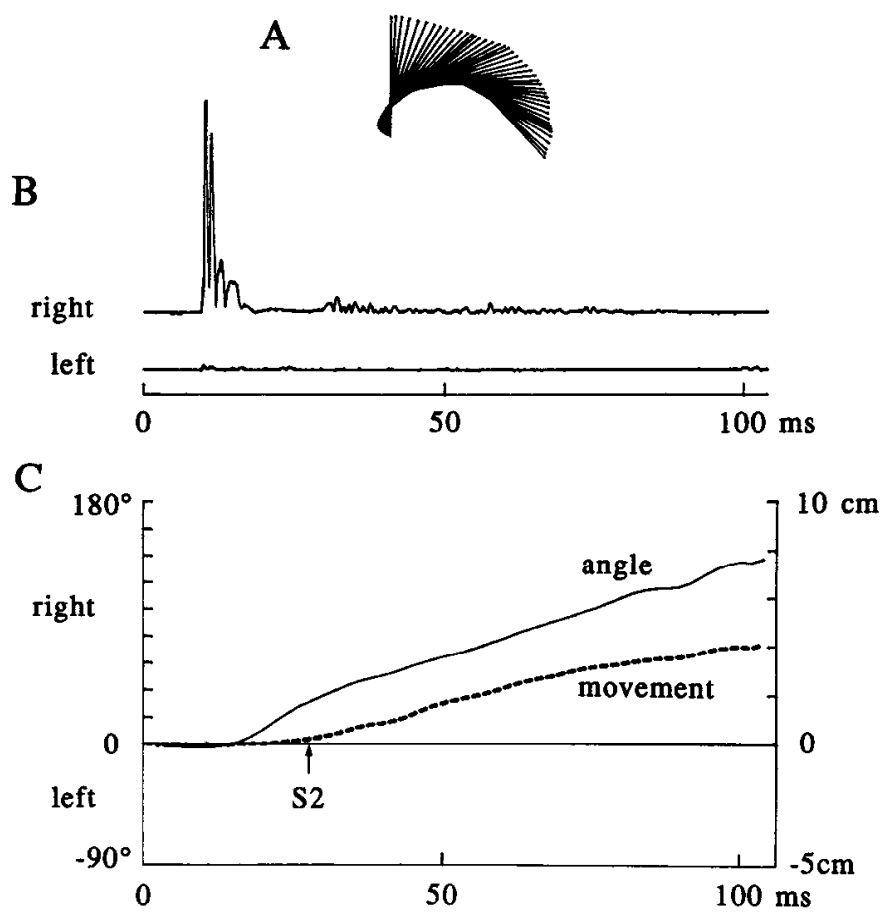

Figure 6. Combined EMG and kinematic data from a response with no DC. $A-C$ are as in Figure 5. This example shows that there is no minor side EMG when there is no DC. Here there is only a major side EMG corresponding to the large unilateral turn. This suggests that no additional contraction is necessary for center of mass displacement

right body musculature (upper trace, Fig. $6 \mathrm{~B}$ ) that matched a large turn to the right. Stage 2 onset (S2, Fig. $6 C$ ) began at 28 msec, but notice that there was no concomitant EMG activity on the minor side (lower trace). Whereas it might be argued that the lack of a minor side EMG was a false-negative failure to record an event that was actually present, Figure 5 shows that we were clearly able to record the minor side EMG when it occurred. (We return to this issue in the Discussion.) Thus, from the example in Figure 6, we see that center of mass propulsion does not require a minor side muscular contraction as revealed by EMG records.

Finally, consider the example in Figure 7, in which there was a DC after stage 2 onset. Here, the minor side EMG occurred $14.4 \mathrm{msec}$ after center of mass propulsion (S2, Fig. $7 \mathrm{C}$ ) and, as in the case in Figure 5, this EMG corresponds temporally to the DC (Fig. $7 C$ ), which occurred $7.3 \mathrm{msec}$ after the onset of the EMG. As in the examples of Figures 5 and 6, there was no minor side EMG associated with stage 2 propulsion.

In sum, from these examples we see that the minor side EMGs temporally correlate with changes in direction, not center of mass propulsion. In cases in which there was no DC, there was no minor side EMG; in cases in which the DC occurred substantially after stage 2 onset, the minor side EMG occurred after the stage 2 onset. Thus, the minor side EMG could not be due to a muscle contraction that causes movement onset. Our findings suggest that, by virtue of a single large contraction on the major side, the fish can execute its propulsive movement, or stage 2 onset. The initial bending of the body and forward propulsion can be due to a unilateral command to the body musculature. Clearly, changes of trajectory require a minor side contraction. This is significant because it suggests that the trajectory is controlled by a bilateral pattern of trunk muscle ac- 

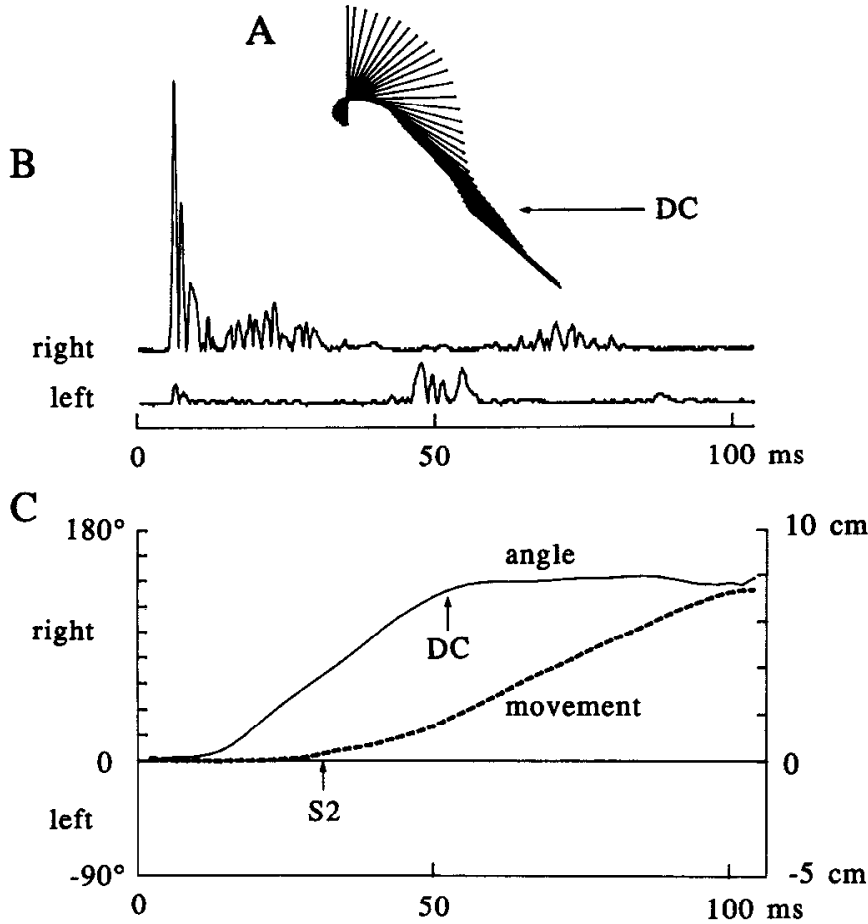

Figure 7. Combined EMG and kinematic data from a response with a late DC. $A-C$ are as in Figure 5. This example shows that the minor side EMG can occur long after the onset of movement and therefore could not be due to a contraction that leads to movement onset. Notice that there is no minor side EMG associated with movement onset ( $S 2$ in $C$ ); rather, the minor side EMG was associated with the DC that occurred at about $50 \mathrm{msec}$ into the response.

tivation that determines the angular components of the animal's movement and does not specifically code forward propulsion. Below, we confirm this quantitatively.

Some important relationships between kinematic and EMG parameters are also suggested by these examples. Notice that the fish in Figures 6 and 7 achieved nearly the same ETA (about $140^{\circ}$ ) in the same amount of time, but did it in different ways. The fish in Figure 7 accelerated faster initially (compare the difference in the slopes of the angle curves of Figs. $6 \mathrm{C}, 7 \mathrm{C}$ ) and slowed its turn by a muscle contraction on the minor side (notice the plateau in the angle curve of Fig. $7 C$ ). In association with this, the major side EMG burst was much larger for the response in Figure 7, which had a higher initial acceleration (compare upper traces in Figs. $6 B, 7 B$ ). This suggests that there are real differences in the EMG magnitude that relate directly to the kinematics and, significantly, that the trajectory at a given point in time can be achieved through a variety of patterns of muscle activation.

In addition to effects reflected by EMG magnitude in these examples, there appear to be effects of EMG timing. In the case of Figure 5, the EMG burst on the minor side occurred relatively soon after the major side burst, and the animal had a correspondingly early DC. In Figure 7, the minor side burst occurred much later, as did the alteration in trajectory. In the next section we ask whether these qualitative effects of magnitude and timing are significant when studied quantitatively.

\section{Magnitude and timing of the EMG bursts correspond to turn angle}

To establish the quantitative relationship between EMG parameters and the angular kinematic components, we first averaged EMG bursts for the major and minor side contractions from the 165 responses of our 14 fish. To do this, our computer program detected the onset of the EMG bursts and synchronized them to a fixed onset time. The data in the panels of Figure 8, $A$ and $B$, were each avcragcd in groups according to four ranges of angles turned, as indicated by the insets. As shown in Figure $8 A$, as stage 1 angle increased from the $0-40^{\circ}$ range to the 80 $100^{\circ}$ range, there was increased EMG activity on the major side. This is qualitatively evident as an increase in burst size, or integral, and also in the size of the first spike in the burst. There was a statistically significant relationship between the integrals

A. Major side EMG increases with stage 1 angle

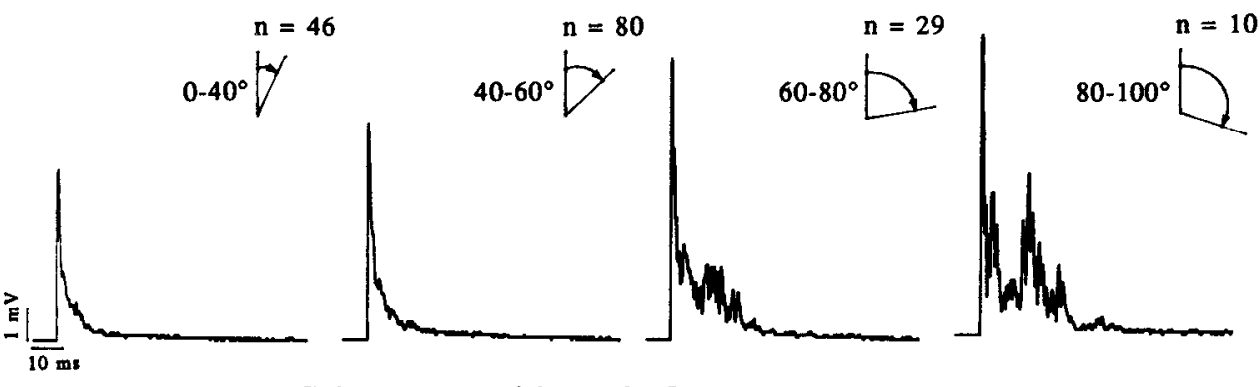

B. Minor side EMG increases with angle 2

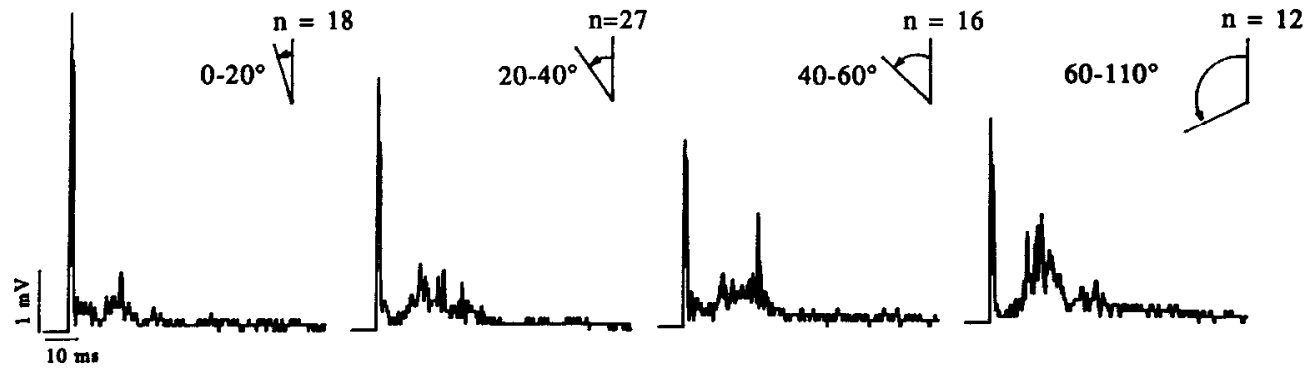

Figure 8. Averaged EMGs showing the relationship between the size of the turn angles and the muscle activity that produces the turns. $A$, Averages of major side EMG activity increased as S1A increased. $B$, Minor side EMG activity increased with $A 2$. Also notice in $A$ that the amplitude of the initial EMG spike increased with increases in turn angle. This relationship was investigated later in a multiple regression analysis and found to be significant (Table 1). To produce these waveforms, the onset times of individual EMG signals were synchronized to a standardized onset time by a computer program. 
Table 1. Multiple regression analysis of the relationship between kinematic and EMG parameters

$$
\begin{aligned}
& \mathrm{S} 1 \mathrm{~A}=1.7(\mathrm{~S} 1 \mathrm{~S} 2)+0.7(\mathrm{LS} 2)-1.6(\mathrm{LS} 1)+41 \\
& A 1=8.9(\mathrm{~S} 1 \mathrm{~S} 2)-8.2(\mathrm{LS} 1)-1.4(\mathrm{LS} 2)-4.0(\mathrm{AS} 1)+173 \\
& \mathrm{DC}=2.6(\mathrm{~S} 1 \mathrm{~S} 2)-2.6(\mathrm{AS} 1)+45 \\
& A 2=5.6(\mathrm{IS} 2)-7.9(\mathrm{AS} 1)+36 \\
& \mathrm{ETA}=9.8(\mathrm{~S} 1 \mathrm{~S} 2)-7.4(\mathrm{LS} 1)-1.8(\mathrm{LS} 2)-4.5(\mathrm{AS} 1)+164
\end{aligned}
$$

$$
\begin{aligned}
& r=0.72, n=85 \\
& r=0.76, n=85 \\
& r=0.58, n=70 \\
& r=0.56, n=50 \\
& r=0.70, n=85
\end{aligned}
$$

$A 1$, angle 1; $A 2$, angle 2; AS1, major side EMG amplitude; DC, time to the change in direction; ETA, apparent escape trajectory angle; IS2, minor side EMG integral; LS1, major side EMG latency; LS2, minor side EMG latency; S1A, stage 1 angle; S1S2, difference between the major and minor side EMG integrals. of these averaged EMGs and the corresponding angles ( $r=0.33$, $p<0.001)$. The integral of the minor side EMG also increased with the extent of the turn during a $\mathrm{DC}$, or $A 2$. As shown in Figure $1 D, A 2$ is the difference between the escape trajectory and the angular heading at the time of the DC. Our analysis of 70 responses with DCs showed that the size of the minor side EMG increased with the size of $A 2$ (Fig. $8 B ; r=0.59, p<$ 0.001 ). The first spike of the EMG bursts in Figure $8 B$ also seemed to decrease with increases in turn angle. There was no significant inverse linear relationship between this spike and turn angle, however.

In sum, these findings from averaged data confirm the fact that our EMG recordings are systematically related to the angular kinematics of the C-start. This suggests that there may be several EMG variables, such as the size of the EMGs and their relative timing, that are functionally related to the kinematics. Therefore, we performed multiple regression analyses by simultaneously regressing individual kinematic variables onto the EMG variables. This allowed a determination of the magnitude of dependence of the kinematic parameters on the EMG parameters. The results of the multiple regression analysis are presented in Table 1. For these analyses, kinematic variables were tested against eight EMG variables. We used SPSS-X (Norusis, 1988 ) to test the EMG variables in a stepwise progression that used a replacement procedure to evaluate the relative contributions of each variable. Table 1 contains five equations for the principle kinematic variables: stage 1 angle (S1A), ETA, $A 1$ and $A 2$, and latency to DC. All of these parameters are depicted in the example of Figure 4.

The regression equations of Table 1 were all highly significant statistically $(p<0.0001)$. We found that the major kinematic parameters could be accounted for in terms of five EMG parameters. For S1 A, ETA, and $A 1$, the most important predictor was S1S2, the difference between the integrals of the EMGs recorded on the major and minor sides. For these angular measures, the next most important predictors were the relative time of onset of the EMG bursts (e.g., LS1, the latency to the major side EMG). The angle produced by a $\mathrm{DC}(A 2)$ was best predicted by the size of the minor side EMG burst (IS2), whereas the time of onset of the DC was best predicted by the difference in EMG integrals between the major and minor sides (S1S2). Another significant parameter was AS1, the amplitude of the major side EMG burst. On average, this was the amplitude of the first EMG spike. This suggests that from the earliest moment of the descending command, motoneurons can be variably recruited depending on the extent of the initial turn (see Discussion).

Our analysis revealed several independent EMG variables that did not contribute significantly to the kinematic parameters. These included the integral of the first small burst on the minor side (indicated by the dot under the lower trace in Fig. $5 B$ ). The initial burst on the minor side is probably a combination of both volume conduction (cross talk) of the EMG signal from the major side and muscle coactivation on the minor side. Due to the large size of the major side EMG, cross talk would be difficult to rule out as an explanation. However, our results suggest that this activity is not due solely to cross talk because it mimics neither the waveform nor the first derivative of the waveform on the opposite side when shown in fast time resolution (data not shown). Bilateral muscle coactivation may contribute to performance by producing body stiffness. However, with our present analysis and recording techniques this small burst made no statistically significant contribution to the kinematics.

\section{Discussion}

This article demonstrates the importance of the angular kinematic components of the descending C-start command that produces the escape trajectory of the fish. We found no evidence for a specific reticulospinal command for forward propulsion (or force) apart from the initial bending of the body. We call this new view the direction change concept and offer it in contrast to our previous biomechanical model (Eaton et al., 1988). With the biomechanical model it was impossible to visualize the C-start movement in the familiar terms of agonist and antagonist muscle contractions. The direction change model is directly analogous to these classical components: during the C-start, the major side contraction may be considered as the agonist and the minor side contraction as the antagonist contraction. Our findings here can be understood in terms of the magnitudes and relative timing of these contractions, or control motions, which we discuss below in the broader context of motor control in vertebrates. Moreover, since forward propulsion can be ignored, the C-start kinematics can be reduced to an analysis of the muscular contractions that cause the simple rotation of the midline, much as has been done previously by others for analyzing rotation of vertebrate limb segments.

\section{Synthesis of the present findings}

In motor control research, it has been difficult to separate kinematic and dynamic motor commands such as force (Soechting and Flanders, 1991), and our previous biomechanical model is an example of this fact. Our present work shows, however, that the force component is embedded in, or is implicit in, the kinematic command that determines the animal's trajectory. This is the rationale for our present emphasis on the angular kinematics or DCs. The biomechanical model was concerned with a description of forces acting on the center of mass. This dynamical concept predicted that there would be a muscular contraction distinct from stage 1 bending and directly resulting in the propulsion of the center of mass (stage 2). We proposed that 
the minor side EMG might underlie center of mass propulsion. However, here we observed that the minor side EMG concurs with DCs and we saw no separate command, or EMG signature, associated with forward propulsion. Rather, forward propulsion is a consequence of the initial turn that is signaled by the stage 1, or major side, EMG burst. By virtue of a single large contraction on one side of the body, the fish can execute a propulsive movement.

This conclusion converges with current theory of how the angular momentum of the initial turn is transferred into the linear momentum that results in fast-start propulsion (for review, see Webb, 1993). The transfer of angular momentum can take place because of the inherent stiffness of the anterior body and the hydrodynamic resistance of the tail. Posterior trunk muscle contractions may contribute to this resistance (Jayne and Lauder, 1993). When the animal's trunk contracts during stage 1, the combination of stiffness and resistance ensures that the tail is bent at an angle relative to the axis of forward motion. This results in a thrust component through the center of mass and causes forward propulsion. Thus, apart from the contraction required to bend the body, a separate propulsive contraction is nol required. This biomechanical theory is based on kinematic data from various studies using different species than our study. Thus, the convergence of our data and this theory adds considerably to the generality of our conclusions.

In concurrence with this, our multiple regression analysis shows that the major and minor side EMG magnitudes and timing accurately reflect the angular kinematic parameters (S1A, ETA, and $A 1$; Table 1). The most significant EMG parameter was the difference between the integrals of the EMGs on the two sides of the body (S1S2). In other words, the angle of the heading according to any of these measures is a result of the difference in strength of bilateral muscular contractions. For example, S1S2 is large when the EMG activity on the agonist side is large relative to the antagonist. In such cases there is a large unilateral turn (Fig. 8). When S1S2 is small, there may be a relatively small agonist EMG burst and a large antagonist EMG burst resulting in a large DC.

Also important was the relative timing of the two bursts on either side of the trunk. In fact, for purposes of discussion, the latency of the major and minor side EMG bursts (LS1 and LS2) can be subtracted to form a single temporal parameter, LS1LS2, equivalent to the interval between the two bursts. The time the DC occurred was a function of the interval between the bursts on both sides, LS1LS2. When this parameter was large, the DC, if any, was late; when it was small, the DC was early.

Initial synchronization of the motoneuronal pool was also a significant factor leading to the $\mathrm{C}$-start, although it was less important than the other EMG parameters. The amplitude of the agonist EMG burst (parameter AS1, Table 1) was significant for three angular measures. As shown in Figure $8 A$, this amplitude parameter is, on average, a measure of the first EMG spike. This short latency spike is associated with the firing of the Mauthner cell (Zottoli, 1977; Eaton et al., 1988; Nissanov et al., 1990), but its size was not previously known to vary systematically with any measure of C-start performance. Although Mauthner cell firing probably underlies part of this potential, the Mauthner cell fires only a single action potential during a response. Clearly, there must be some synchronized motoneuron firing that accompanies the progressively larger EMG signal that occurs with larger response angles. One possibility is that other reticulospinal neurons can be nearly co- active with Mauthner cell firing and are recruited at progressively larger response angles.

To summarize, the C-start escape trajectories can be accounted for by the relative magnitude and timing of a bilateral pattern of trunk muscle contractions that determine the angular components of the escape trajectory. Thus, escape trajectory is a function of the magnitude of two control motions generated by the agonist (major side) and antagonist (minor side) muscle contractions and their relative onset times. These two motions provide initial acceleration and braking, or trajectory modification.

These conclusions are important because they unify the diversity of C-start movement patterns, such as those seen in Figure 2, into a single and rather simple conceptual scheme. In the absence of our EMG analyses, one might be tempted to categorize $\mathrm{C}$-start kinematics into a dictionary of different response "types," and then to search for reticulospinal neurons whose activity patterns reflect the different types of responses. In fact, as discussed by Webb (1993), this temptation has already led to a burgeoning terminology in the literature on C-start biomechanics. In contrast, our findings greatly simplify the notion of what a C-start is and how it can be analyzed to understand its underlying neural basis. Response characterization according to diverse patterns of acceleration or other physical parameters is important for many biomechanical and functional studies. For our purposes, however, rather than categorizing a wide variety of response types, one can think of the behavior in terms of only a few kinematic parameters that characterize the magnitude and timing of the muscle contractions that are the result of the reticulospinal commands.

\section{Comparison with vertebrate limb movements}

Our findings are analogous to the systematic changes in EMG amplitude and timing that occur in limb movements. For instance, in the experiments of Sherwood et al. (1988), human subjects performed a rapid forearm flexion and extension movement. Such motions are comparable to $\mathrm{C}$-starts in which there is a major DC. For the humans, movement time was kept constant while amplitude (or joint angle) was varied. As with C-starts having DCs, agonist EMG size and antagonist EMG size were found to increase systematically with joint angle. As for the C-start, when the time to a DC was the same, there did not appear to be changes in the relative EMG onset times in humans (e.g., Fig. 4 of Sherwood et al., 1988).

In related experiments on humans, movement time was increased while amplitude was held constant, and the EMG burst onset time of the antagonist increased (e.g., Fig. 4 of Schmidt et al., 1988). It would be especially illuminating to compare this finding with an analysis of fish C-starts in which EMG integrals and timing are analyzed for responses (such as in Figs. 6, 7) in which there are similar response angles achieved in different intervals of time. Our analyses were not designed with this in mind, but the prediction from our findings is that the goldfish would show a similar pattern.

The comparison between trunk and limb movements is not upset by the fact that the C-start always involves forward propulsion. Propulsion translates the rotation over distance rather than simply pivoting the fish about a single point. This might seem to provide an additional degree of freedom in the fish movement that is not present in the experiments on humans, but we saw no separate EMG command corresponding to stage 2 onset. Although C-start distance performance can vary from 
trial to trial, there is no systematic relationship between the distance moved and the ETAs (Eaton et al., 1988; Webb, 1993). This is evident in the examples in Figure 2, in which the lengths of the arrows correspond to the distance moved by the center of mass-for these 11 cases, an average of $4.9 \mathrm{~cm}$. Thus, although there is a translational movement of the C-start rotation, an important simplifying fact is that regardless of the angle turned by the fish, its propulsion is maximized and the distance moved in a given interval is relatively constant. We have shown that larger response angles involve progressively more motoneuron activation, but the additional force generated is dissipated by the larger turn. This results in responses of approximately the same distance moved by the center of mass (for further discussion, see Webb, 1993).

Given that forward propulsion can be disregarded, an important feature of the direction change model is that, like the rapid limb movement control, the fish appears to utilize the mechanical properties of the muscle, thus reducing the neurocomputational demands of movement planning. Various mechanical analogs have been proposed in which the limb segment can be likened to a rod on a pivot whose angle is controlled by two opposing springs or forces controlled by the motor command (Polit and Bizzi, 1978; Doonskaya, 1992). Our data suggest that rotation of the fish midline can be thought of in similar terms, though we are not yet prepared to specify a particular analog. The similarity is important because by specifying the contractions and timing of the opposing muscles, the fish can quickly specify trajectories from a few simple parameters without having to rely on feedback measurements of position. This simplifies the neurocomputational problem for the fish nervous systcm and probably reduces the time to generate an escape response.

This model also makes sense because during the C-start, the animal undergoes a very rapid angular and linear acceleration $(5 \times g$ or more; Harper and Blake, 1990; Domenici and Blake, 1991; Webb, 1993). One has to wonder what useful sensory information from any modality could be provided during this displacement. This is not to say that all sensory feedback is ignored, only that feedback may be relatively unimportant during the early movements of the behavior when the gross details of the trajectory are being specified. In fact, several lines of evidence already support the notion that the C-start in the goldfish is an open-loop behavior. For example, the fish does not appear to correct its trajectory if it makes an apparent "error" and turns toward the aversive stimulus (Eaton and Emberley, 1991).

\section{Conceptual summary}

Body muscle contractions during the C-start are produced by a corresponding set of descending reticulospinal commands that are ultimately a reflection of the perceived direction of the aversive stimulus. The summary diagram in Figure 9 incorporates both our recent work on the directional production of the C-start (Eaton and Emberley, 1991) and the EMG and kinematic findings in the present study. This diagram represents a working hypothesis to guide future exploration of the neurophysiology of the identified reticulospinal neurons. The sensorimotor process is visualized in terms of both crossed and uncrossed neurons in the brainstem escape network, as shown in Figure $9 A$. The $A 1$ cells activate the agonist contraction and $A 2$ cells activate antagonist muscle contractions. Both populations act in concert to determine the complete variety of trajectories. These trajec- tories are produced by the relative activation and timing of the two populations. For the purposes of the diagram we assume that the agonist contraction may be triggered at least in part by the Mauthner series homologs (Eaton et al., 1991; Lee and Eaton, 1991; Lee et al., 1993) with axons that cross to the opposite side of the spinal cord. The antagonist contraction is visualized as being controlled by cells with axons that do not cross. This feature is not essential but it is based on the parsimonious assumption that the cells controlling the behavior may be located primarily on the side of the brain closest to the stimulus.

To visualize how such a network could function, we present in Figure $9 B-D$ three different examples of responses to stimuli from three directions: caudal, lateral, and rostral. Turns involving significant bilateral muscle contractions (Fig. 9B) are produccd in responsc to stimuli approaching the fish from behind (Eaton and Emberley, 1991). This minimally activates the $A 1$ neurons (Fig. 9BI) and maximally activates the antagonists (Fig. 9B2). Accordingly, agonist EMGs for such turns are relatively smaller and antagonist EMGs are relatively larger. Unilateral turns (Fig. 9D) are produced in response to stimuli approaching the fish from the front (Eaton and Emberley, 1991). Such turns involve a single large EMG agonist burst with little or no antagonist activity. Thus, we can imagine that rostral stimuli maximally activate the $A 1$ cells, as shown in Figure $9 D 1$, to produce enhanced motoneuronal firing, whereas the antagonist neurons (Fig. 9D2) are minimally activated by this stimulus. Figure $9 C$ is an intermediate case. Except for the inclusion of the Mauthner cell, this hypothetical network is not intended to have a literal interpretation. Rather, it may be used to visualize how the muscle contractions we have observed might be controlled by populations of reticulospinal ncurons.

We can quantitatively describe the input-output relationship for the portion of the model giving rise to the initial orientation, S1A. The magnitude of the turn during stage 1 (S1 A) is a linear function of the angle of the aversive stimulus (stimulus angle, $\mathrm{SA}$; Fig. $1 C$ ) relative to the center of mass (Eaton and Emberley, 1991):

$$
\mathrm{S} 1 \mathrm{~A}=0.33 \mathrm{SA}-113^{\circ} .
$$

In effect, Equation 3 describes the overall input-output relationship of the sensorimotor process leading to the initial orientation. The present study gives a quantitative basis to the turn during stage 1 in terms of the underlying EMG signals, which are a function of the descending commands of the brainstem escape network (for simplicity we omitted the coefficients):

$$
\text { S1A = S1S2 - LS1SL2 + constant. }
$$

By combining Equations 3 and 4 we derive the overall relationship between the agonist and antagonist EMG magnitude and timing parameters as a function of the angle of the impinging stimulus:

$$
\text { S1S2 - LS1LS2 = 0.33SA + constant. }
$$

This equation accounts for the pattern of EMG activity during the initial orientation of the C-start in terms of the angle of the aversive stimulus relative to the body of the fish. It is important to note that for any SA there are a variety of EMG magnitudes and timing patterns. This may seem untidy, but it accurately represents the variety of ways that the fish produces its orientations. Equation 5 should prove to be very useful in explorations of the neurophysiology of the reticulospinal neurons belonging to the brainstem escape network. 


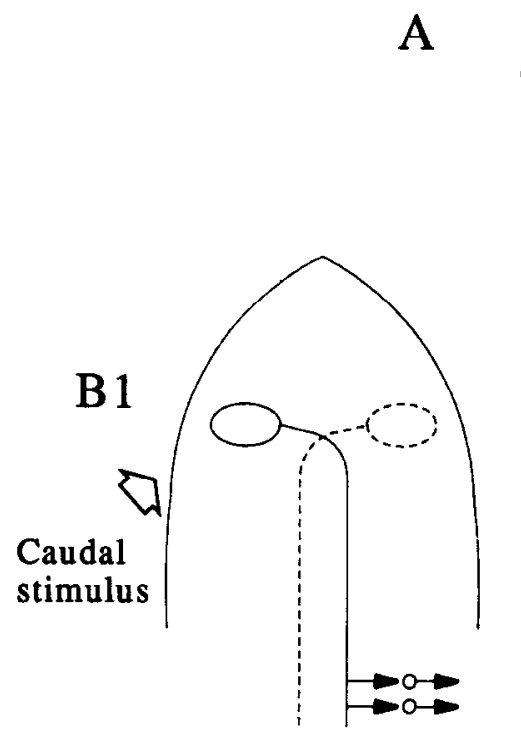

C1

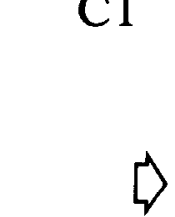

Lateral stimulus
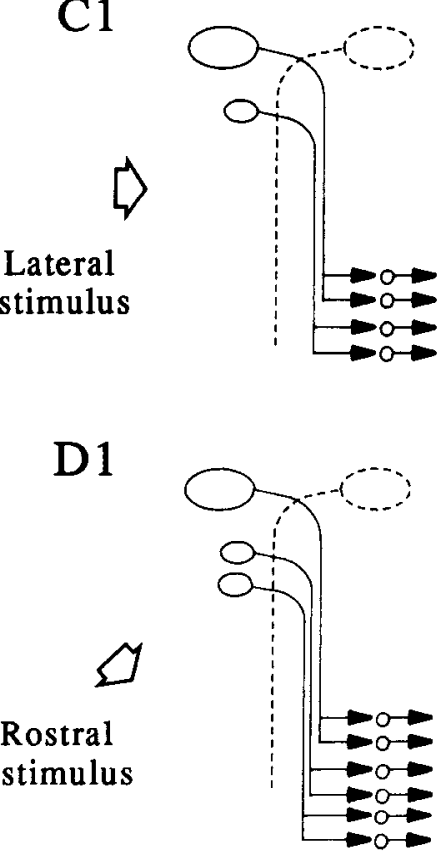

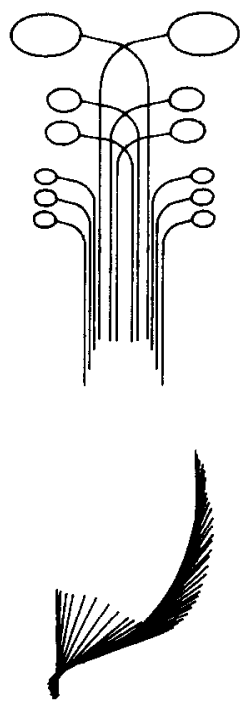

A1 neurons

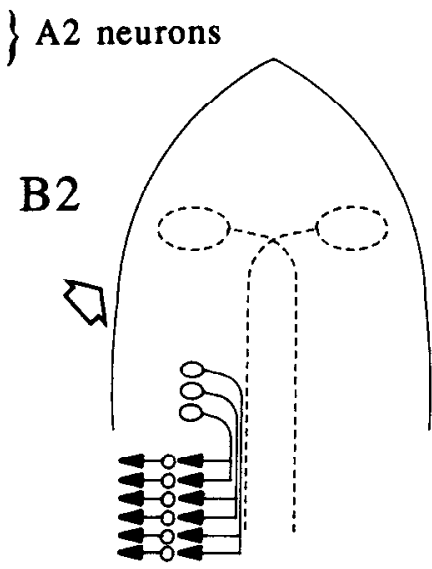

$\mathrm{C} 2$

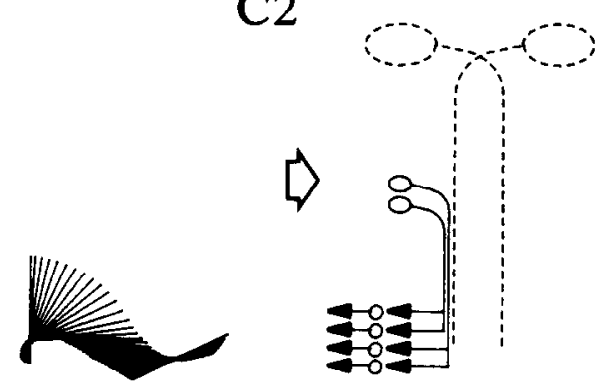

D2

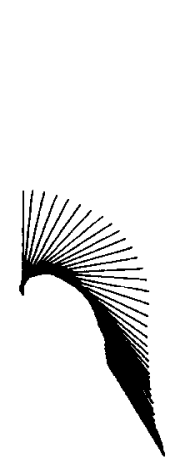

Figure 9. Summary of possible sensorimotor interactions leading to the various patterns of escape responses. $A$, The model is based on two kinds of neurons in the brainstem escape network $(A 1$ and $A 2)$ that activate spinal motoneurons and result in the agonist and antagonist contractions. In this model, the brainstem cells can be differentially recruited and activated at different intervals after the stimulus to produce various escape trajectories. $B$, An example similar to the response in Figure 5 . Here a caudal stimulus minimally activates the $A 1$ neurons, causing a small agonist contraction $(B 1)$, and maximally activates the $A 2$ neurons, resulting in a large antagonist contraction (B2). C, Example with the stimulus from the side. Here there is a relatively more even balance between excitation of the $A 1$ and $A 2$ neurons. $D$, A case similar to Figure 6 , in which a rostral stimulus maximally activates the $A 1$ neurons to produce an agonist contraction that reverses the orientation of the fish. This stimulus only minimally recruits $A 2$ neurons.
An ultimate goal of this work is to describe the quantitative relationship between the SA and resulting ETA in a way similar to that for Equation 5, but this would be premature using the present data. First, we are uncertain how much the walls of the test arena may have influenced the values for ETA from our earlier study (Eaton and Emberley, 1991). We know that when fish were close to a wall at the start of the response, escape trajectories were altered significantly (Eaton and Emberley, 1991). The previously observed variability in ETA values may have been increased by these effects. Second, during responses such as in Figure 3, the fish was still turning at the time the escape trajectory was measured. Continued turning may also be an influence of the walls of the aquarium. Third, the ETA is a function of $A 2$ (Eq. 1), but we do not yet know the relationship between $A 2$ and SA. All these issues could be readily resolved by performing experiments similar to the present ones, but in- stead using a known directional stimulus source in a much larger arena and over a longer analysis interval. Such an experiment would also resolve uncertainties about the kinematic transition between the C-start and the onset of steady swimming following the initial fast-start movements.

\section{C-start parameters}

Our direction change concept prompts the question as to what are the most relevant biomechanical or kinematic measures of the C-start. Is $A 1$ or $\mathrm{S} 1 \mathrm{~A}$ the most appropriate measure of the initial angular component of the C-start? Provisionally, we have focused on S1A because it has a closer temporal relationship to the EMG burst on the major side. $A 1$ is determined at a point in time that is either the same, or later than, the time at which $\mathrm{S} 1 \mathrm{~A}$ is measured (therefore, it is always true that $A 1 \geq \mathrm{S} 1 \mathrm{~A}$; Figs. $1, C$ vs $D ; 4 C$ ). Although we believe that $A 1$ is the ap- 
propriate descriptor of the first turn leading to the FTA, one needs to consider EMG parameters such as the antagonist EMG and its onset time to account for $A 1$. When we have a better mathematical characterization of these complexities, S1 A may be dropped from usage. However, because it occurs earlier in time, SlA is a more conservative measure of the consequences of the agonist EMG when we do not take other factors into account.

We originally introduced stage 2 angle as a way to measure the variability of the turning movements that occur after stage 1 (Eaton et al., 1988). However, stage 2 angle is not a useful parameter in the context of the present experiments because it has no straightforward relationship to the underlying pattern of muscular contractions. $A 2$ is always a single rotational movement (in one direction), whereas stage 2 angle can include a DC and therefore does not necessarily represent a unilateral command to the trunk musculature (Figs. $1, C$ vs $D ; 4 C$ ).

What about stage 2 onset, which is related to the movement of the center of mass? Although we show that this parameter is not separately specified by a reticulospinal command, it should continue to be important for biomechanical studies of the underlying forces that produce the behavior; moreover, stage 2 onset may be an important measure of the success of the behavior, since predators may target the center of mass (Weihs and Webb, 1984). In sum, it appears that selection of such parameters will depend on the application and form of the analysis.

\section{The role of red muscle}

In a recent study Jayne and Lauder (1993) reported that both red and white muscles were synchronously activated during both stages of the C-start. This is a surprising result because red muscle was previously thought to be involved only in the forward propulsion of steady swimming at moderate velocities, and not in the high-performance maneuvers of C-starts (Rome and Sosnicki, 1991). Our wide-field EMG electrodes were intended to record white muscle activity and may not have detected red muscle activity. Thus, one counterargument to our main thesis is that red muscle, which we did not specifically record, was responsible for stage 2 propulsion. However, even though red muscle can be coactive with the white during C-starts, it is unlikely that stage 2 propulsion would be due only to the red muscle fibers while the white muscle was silent. This notion is inconsistent with both C-start biomechanics discussed earlier and with red muscle physiology. Forward propulsion begins on average about $25 \mathrm{msec}$ after the start of the stage 1 rotation (Eaton et al., 1988), yet the red muscle fibers take approximately $45 \mathrm{msec}$ to reach peak tension whereas the white reach peak tension in about 11-18 msec (see data cited by Jayne and Lauder, 1993). The function of the behavior is to displace the animal as rapidly as possible from its initial location (Eaton et al., 1991). Given their slow mechanics, it is illogical to think that red fibers by themselves would mediate C-start propulsion while the white anlagonists, which contract three times faster, remained silent.

\section{Conclusion}

The neural mechanisms underlying the fish escape response may represent a fundamental reticulospinal program used even in cases where the anatomical organization of the musculature is quite different. There are several reasons for thinking this. First, the reticulospinal system is highly conserved over the course of evolution (see reports cited in Nissanov and Eaton, 1989). Sec- ond, C-start movements occur very early in development (Eaton and DiDomenico, 1985). Third, the brainstem appears to be organized on a segmental pattern whose development is regulated by common mechanisms involving the Hox/homeobox genes (Lumsden and Keynes, 1989; Wilkinson et al., 1989; Kessel and Gruss, 1990). Finally, lateral bending of the trunk, as during the fish escape response, is believed to be a primitive vertebrate motor pattern (Eaton, 1992; Golani, 1992). Thus, given the apparent similarity of EMG patterns in systems as diverse as the fish trunk and human arm, it could be that our findings will have implications for understanding many kinds of rapid movements. For instance, a common motor pattern generator may be used for different locomotor behaviors at different stages of development (Bekoff, 1991). Likewise, a fundamental reticulospinal program may play a role in the apparent "economy" of motor systems, noted by Schmidt et al. (1988), where diverse kinds of movements might be produced by straightforward modifications of motor control.

For uncovering neurophysiological foundations of such a mechanism at the level of the brainstem, the fish preparation should prove to be useful because of the relative simplicity of its neural networks and the bilateral symmetry of the trunk musculature that produces the agonist and antagonist contractions. In addition, the C-start is an involuntary escape response and the angular movement parameters, which determine where the fish goes, are among the most important factors in determining whether the animal successfully avoids a predator (Weihs and Webb, 1984). These parameters are likely to have been strongly shaped by natural selection and are therefore especially relevant to understanding basic mechanisms of motor control.

\section{References}

Bekoff AC (1991) Neuroethological approaches to the study of motor development in chicks: achievements and challenges. J Neurobiol 23: 1486-1505.

Bizzi E, Mussa-Ivaldi A (1989) Geometrical and mechanical issues in movement planning and control. In: Foundations of cognitive science (Posner MI, ed), pp 769-792. Cambridge: MIT Press.

Cohen AH (1992) The role of heterarchial control in the evolution of central pattern generators. Brain Behav Fvol 40:112-124.

Dominici P, Blake RW (1991) The kinematics and performance of the escape in the angelfish (Pterophyllum eimekei). J Exp Biol 156: $187-205$.

Doonskaya NV (1992) The common control activity pattern providing for fast movements. In: Proceedings of the third international congress of neuroethology, abstr 312. Montreal: McGill UP.

Eaton RC (1992) Eshkol-Wachman movement notation and the evolution of locomotor patterns in vertebrates. Behav Brain Sci 15:272274.

Eaton RC, DiDomenico R (1985) The role of the teleost escape response during development. Trans Am Fish Soc 115:128-142.

Eaton RC, Emberley DS (1991) How stimulus direction determines the trajectory angle of the Mauthner initiated escape response in a teleost fish. J Exp Biol 161:469-487.

Eaton RC, Lavender WA, Wieland CM (1981) Identification of Mauthner initiated response patterns in goldfish: evidence from simultaneous cinematography and electrophysiology. J Comp Physiol [A] 144:521-531.

Eaton RC, DiDomenico R, Nissanov J (1988) Flexible body dynamics of the goldfish C-start: implications for reticulospinal command mechanisms. J Neurosci 8:2758-2768.

Eaton RC, Nissanov J, DiDomenico R (1991) The role of the Mauthner cell in sensorimotor integration by the brainstem escape network. Brain Behav Evol 37:272-285.

Evarts EV (1966) Pyramidal tract activity associated with a conditioned hand movement in the monkey. J Neurophysiol 29:10111027. 
Faber DS, Korn H, Lin J-W (1991) The role of medullary networks and postsynaptic membranc propertics in regulating Mauthncr cell responsiveness to sensory excitation. Brain Behav Evol 37:286-297.

Feldman AG (1986) Once more on the equilibrium-point hypothesis ( $\lambda$ model) for motor control. J Motor Behav 18:17-54.

Fetcho JR (1991) The spinal network of the Mauthner cell. Brain Behav Evol 37:298-316.

Fetz EE (1992) Are movement parameters recognizably coded in the activity of single neurons? Behav Brain Sci 15:679-690.

Foreman MB, Eaton RC (1990) EMG and kinematic analysis of the stages of the Mauthner-initiated escape response. Soc Neurosci Abstr $16: 1328$.

Golani I (1992) A mobility gradient in the organization of vertebrate movement: the perception of movement through symbolic language. Behav Brain Sci 15:249-308.

Gottlicb GL, Corcos DM, Agarwal GC (1989) Stratcgics for the control of voluntary movements with one mechanical degree of freedom. Behav Brain Sci 12:189-250.

Harper DG, Blake RW (1990) Fast-start performance of rainbow trout (Salmo gairdneri) and northern pike (Esox lucius). J Exp Biol 150: 321-342.

Jayne BC, Lauder GV (1993) Red and white muscle activity and kinematics of the escape response of the bluegill sunfish during swimming. J Comp Physiol [A], in press.

Kessel M, Gruss P (1990) Murine development control genes. Science 249:374-379.

Lauder GV (1983) Food capture. In: Fish biomechanics (Web6 PW, Weihs D, eds), pp 280-311. New York: Praeger.

Lee RKK, Eaton RC (1991) Identifiable reticulospinal neurons of the adult zcbrafish, Brachydanio rerio. J Comp Ncurol 304:43-52.

Lee RKK, Eaton RC, Zottoli SJ (1993) Segmental arrangement of reticulospinal neurons in the goldfish hindbrain. J Comp Neurol 327: $1-18$.

Loeb GE, Gans C (1986) Electromyography for experimentalists. Chicago, IL: University of Chicago.

Lumsden A, Keynes R (1989) Segmental patterns of neuronal development in the chick hindbrain. Nature 337:424-428.

McClellan AD (1986) Command system for initiating locomotion in fish and amphibians parallels to initiation systems in mammals. In: Neurobiology of vertebrate locomotion (Grillner S, Stein PSG, Stuart DG, Forssberg H, Herman RM, eds), pp 3-20. London: Macmillan.

Metcalfe WK, Mendelson B, Kimmel CB (1986) Segmental homologies among reticulospinal neurons in the hindbrain of the zebrafish larva. J Comp Neurol 251:147-159.

Nissanov J, Eaton RC (1989) Reticulospinal control of rapid escape turning in fishes. Am Zool 29:103-121.

Nissanov J, Eaton RC, DiDomenico R (1990) The motor output of the Mauthner cell, a reticulospinal command neuron. Brain Res 517: 88-98.
Norusis MJ (1988) SPSS-X advanced statistics guide, 2d ed. Gorinchem: SPSS International BV.

Peterson BW (1984) The reticulospinal system and its role in the control of movement. In: Brain stem control of spinal cord function (Barnes CD, ed), pp 27-86. New York: Academic.

Polit A, Bizzi E (1978) Processes controlling arm movements in monkeys. Science 210:1235-1237.

Rome LC, Sosnicki AA (1991) Myofilament overlap in swimming carp. II. Sarcomere length changes during swimming. Am J Physiol 247:R272-R279.

Schmidt RA, Sherwood DE, Walter CB (1988) Rapid movements with reversals in direction. I. Control of movement time. Exp Brain Res 69:344-354

Sherwood DE, Schmidt RA, Walter CB (1988) Rapid movements with reversals in direction. II. Control of movement amplitude and inertial load. Exp Brain Res 69:355-367.

Soechting JF, Flanders M (1991) Deducing central algorithms of arm movement control from kinematics. In: Motor control: concepts and issues (Humphrey DR, Freund H-J, eds), pp 293-306. New York: Wiley.

Stein RB (1982) What muscle variable(s) does the nervous system control in limb movements? Behav Brain Sci 5:535-577.

Wainwright PC (1983) To bend a fish. In: Fish biomechanics (Webb PW, Weihs D, eds), pp 68-91. New York: Praeger.

Wainwright PC (1989) Prey processing in haemulid fishes: patterns of variation in pharyngeal jaw muscle activity. J Exp Biol 141:359-375.

Wallace SA (1981) An impulse-timing theory for reciprocal control of muscular activity in rapid, discrete movements. J Motor Behav $13: 144-160$

Wcbb PW (1978) Fast start performance and body form in seven species of teleost fish. J Exp Biol 74:211-226.

Webb PW (1993) Exercise performance of fish. In: Comparative vertebrate exercise physiology (Jones $\mathrm{JH}$, ed), in press. Orlando, FL: Academic.

Webb PW, Skadsen JM (1980) Strike tactics of Esox. Can J Zool 58: 1462-1469.

Weeks DL, Sherwood DE, Wallace SA (1991) EMG area scaling and velocity modulations in a spatiotemporally constrained movement. J Electromyogr Kines 1:250-262.

Weihs D, Webb PW (1984) Optimal avoidance and evasion tactics in predator-prey interactions. J Theor Biol 106:189-206.

Wilkinson DG, Bhatt S, Cook M, Bonicelli E, Krumlauf R (1989) Segmental expression of Hox-2 homeobox-containing genes in the developing mousc hindbrain. Naturc 341:405-409.

Williams T, Grillner S, Smoljaninov VV, Wallén P, Kashin S, Rossignol $S$ (1989) Locomotion in lamprey and trout: the relative timing of activation and movement. J Exp Biol 143:559-566.

Zottoli SJ (1977) Correlation of the startle reflex and Mauthner cell auditory responses in unrestrained goldfish. J Exp Biol 66:243-254. 